\title{
Italique
}

Poésie italienne de la Renaissance

XVIII | 2015

Varia

\section{Dallo scrittoio al teatro: considerazioni sulle tragedie giraldiane}

Susanna Villari

\section{(2) OpenEdition}

1 Journals

Edizione digitale

URL: http://journals.openedition.org/italique/401

DOI: 10.4000/italique.401

ISSN: 1663-4438

\section{Editore}

Librairie Droz

\section{Edizione cartacea}

Data di pubblicazione: 1 octobre 2015

Paginazione: 13-34

ISBN: 978-2-600-01900-2

ISSN: 1423-3983

Notizia bibliografica digitale

Susanna Villari, « Dallo scrittoio al teatro: considerazioni sulle tragedie giraldiane », Italique [Online], XVIII | 2015, online dal 01 octobre 2017, consultato il 21 décembre 2020. URL : http://

journals.openedition.org/italique/401; DOI : https://doi.org/10.4000/italique.401

(c) Tous droits réservés 


$$
\text { S U S A N A VILLAR I }
$$

D A L L O S C R I T T O I O A L T E A T R O: C O N S D E R A Z I O N I S L LE TRA GEDIE GIR A L D I N E 



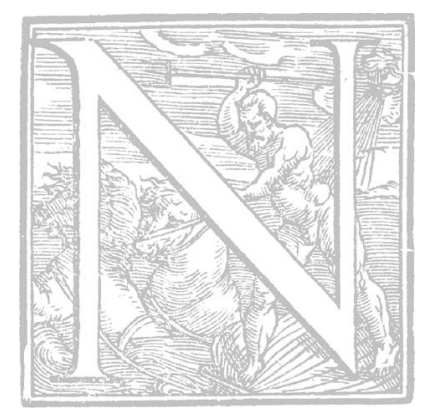

el 1963, nella sua recensione al libro di Horne sulle tragedie di Giraldi Cinzio, Dionisotti ${ }^{\mathrm{I}}$ propose agli studiosi una questione rimasta tuttora aperta, relativa alle ragioni per le quali una sola delle nove tragedie giraldiane, l'Orbecche, $f u$ pubblicata vivente l'autore ed ebbe grande fortuna:

uno studio critico sulle tragedie del Giraldi dovrà sì, come in questo libro [di Horne] è stato egregiamente fatto, procedere a un imparziale esame di tutte e nove le tragedie, ma anche e anzitutto dovrà accettare e spiegare il fatto che tra le nove soltanto l'Orbecche ebbe una qualche importanza nella storia della letteratura italiana. La quale storia, piaccia o non piaccia, è quel che è, non è storia modificabile per nostro gusto o capriccio, anche se sia col tempo modificabile per nostro ingegno e fatica. Nel caso di Giraldi, è chiaro che, prima di render conto dell'insuccesso di quelle sue tragedie ai lettori del tardo Cinquecento e dei secoli successivi fino al nostro, bisognerà chieder conto al Giraldi stesso del fatto che, dopo aver pubblicato l'Orbecche, egli ritenne di non poter o dover pubblicare o comunque divulgare le altre sue tragedie. Poiché nel i 554 egli invece pubblicò il suo Discorso intorno al comporre delle comedie e delle tragedie, mi pare si possa concludere che, al di là del contrastato ma indubbio successo dell'Orbecche, il Giraldi decise che era per lui più utile e meno rischioso discutere in prosa sul teatro che non dare in versi altre pubbliche prove, fuori di Ferrara, del suo scarso talento di poeta di teatro. ${ }^{2}$

Mentre Horne distoglieva $i$ critici dall'esclusiva considerazione dell'Orbecche (frutto di una poetica tragica dell'orrore di matrice senecana), in nome di una complessiva valutazione del teatro tragico giraldiano (che fa registrare il distacco da quella tipologia di tragedia con epilogo cruento e terrificante), Dionisotti richiamava l'attenzione sulla circostanza storica della particolare fortuna dell'Orbecche $e$ sulla scelta giraldiana di lasciare inedite le altre tragedie. Il mutamento di prospettiva critica attuato da Horne, ovvero il superamento dell'angusta concezione di un Giraldi poeta dell'orrido, non avrebbe dovuto, secondo Dionisotti, far perdere di vista il fatto che forse Giraldi stesso 
non fosse convinto della validità di quelle tragedie rimastegli tutta la vita nel cassetto. ${ }^{3}$

Questa conclusione estrema (temperata dall'invito a più accurate verifiche critico-filologiche e a un esame più specifico del contesto storicoculturale degli anni, I540-I563, in cui si colloca la sperimentazione tragica giraldiana), può essere assunta oggi come base di partenza per rilanciare il quesito, alla luce di una critica giraldiana più agguerrita e matura e sulla scorta delle edizioni moderne dei testi tragici: Giraldi era davvero incerto dei risultati raggiunti? Perché, dopo il successo ottenuto con l'Orbecche, egli non pubblicò le altre sue tragedie?

Una prima riflessione può scaturire certamente dalle articolazioni del dibattito sul genere tragico e dalle accese polemiche che si svilupparono a partire dagli anni Quaranta del secolo, ${ }^{4}$ proprio in concomitanza con la divulgazione dell'Orbecche giraldiana, di cui la Canace di Sperone Speroni si presentò come immediata antagonista. ${ }^{5}$ Le resistenze erudite nei confronti di scelte poetiche innovative, non giustificabili entro le coordinate dei precetti desunti dalla Poetica aristotelica, furono notevoli e investirono dunque la prima tragedia giraldiana, che, "fuor del costume / delle tragedie e de' poeti antichis,, si offriva al pubblico caratterizzata da un soggetto di nuova invenzione, dal prologo separato (in uso nelle commedie latine), dalla divisione in atti e in scene, dal suicidio della protagonista a scena aperta e dalla ricerca di un effetto naturale del linguaggio. ${ }^{7}$ Gli attacchi dei detrattori non ostacolarono tuttavia la diffusione dell'Orbecche, pubblicata varie volte mentre l'autore fu in vita. Ciò conferma anzitutto che non furono le critiche accademiche a scoraggiare Giraldi, che nel corso della sua carriera di tragediografo difese strenuamente la propria strategia innovativa, convinto della necessità di una scrittura idonea alle esigenze dei tempi e alle finalità pedagogiche. Del resto, la corte ferrarese di Ercole II d'Este, e poi di Alfonso II, costituiva un terreno favorevole alla sperimentazione teatrale: Giraldi ebbe il ruolo di tragediografo di corte e operò con grande libertà nel proporre, accanto a soggetti teatrali desunti dal mito e dalla storia (le tragedie Didone e Cleopatra), fabulae fictae, ricavate dalla sua raccolta novellistica (Gli Ecatommiti), che, almeno fino al 1563 , era in fase di allestimento. ${ }^{8}$ Ad eccezione dell'Epizia, che, secondo la testimonianza di Celso Giraldi, non fu mai rappresentata, ${ }^{9}$ sappiamo che le altre tragedie furono invece messe in scena 
con successo. ${ }^{\circ}$ Giraldi aveva avuto modo, dunque, di sperimentare l'impatto etico-pedagogico dei suoi testi e di affinare la propria concezione di un teatro moderno che consentisse di veicolare problematiche filosofiche, giuridiche, politiche, etiche e offrirsi come luogo di riflessione e di rinnovamento civile e morale.

Le ragioni della mancata pubblicazione della maggioranza delle tragedie (e per l'Epizia pure di una mancata rappresentazione) non possono semplicisticamente ricondursi a un'ostilità del mondo accademico (se cosi fosse, l'Orbecche non avrebbe avuto tanta fortuna), ma neppure a un'idea giraldiana di scarsa «validità di quelle tragedie» (Dionisotti), perché non si spiegherebbe altrimenti il notevole impegno teorico con cui Giraldi, nel corso di tutta la propria carriera poetica, accompagnò la sperimentazione teatrale e volle difendere le proprie scelte. La verità sta forse in mezzo, e va ricercata nel contesto degli scritti giraldiani, ripercorrendo i momenti più significativi della speculazione poetica dell'umanista ferrarese. Non risolutiva ai fini di una risposta al nostro quesito è l'osservazione (fondamentale per caratterizzare l'itinerario drammaturgico giraldiano) che l'esperienza tragica giraldiana fu tutta «interamente e schiettamente scenicas: ${ }^{.1}$ anche il successo dellOrbecche si realizzò anzitutto sulla scena, quando Giraldi allesti a casa propria (con l'apporto di attori professionisti, scenografi e musicisti) una prima rappresentazione della tragedia, alla quale ne seguirono altre, su sollecitazione delle persone illustri che avevano assistito a quel primo spettacolo. ${ }^{\mathrm{I} 2}$ Ma ciò non distolse l'autore da una pubblicazione della tragedia, per soddisfare coloro che l'avevano apprezzata. ${ }^{3}$ Anzi è esplicito, già nel I543, il proposito giraldiano di pubblicare, traendo auspicio dalla buona accoglienza dell'Orbecche, anche le altre tre tragedie fino a quel momento composte:

E s'ella [l'Orbecche] fia da voi [Ercole II d'Este] con quello animo accolta con cui la vostra rara virtude e molta cortesia mi promette che serà, io non dubito ch'ella non rimanga da ogn'invidia sicura [...]. Il che, se fia, si darà ardire alle altre sue sorelle, Altile, Cleopatra e Didone, ch'ora timide appresso di me stanno nascose, di lasciarsi vedere. ${ }^{14}$

Il consenso del duca non mancò, come si evince non soltanto dalle parole di Giraldi stesso nella Lettera in difesa della Didone, ${ }^{\mathrm{I}}$ ma anche dal fatto che Ercole II d'Este continuò a commissionare a Giraldi l'elaborazione e rappresentazione di tragedie. ${ }^{16}$ 
Non va inoltre dimenticato che un progetto di divulgazione a stampa delle tragedie giraldiane era ancora vivo a distanza di oltre un ventennio, nel I565, come attestano le parole di Arlenio Arnoldo, nell'epistola ai lettori edita in calce agli Ecatommiti, laddove egli dichiarava che la favorevole accoglienza della raccolta giraldiana lo avrebbe incoraggiato a breve a stampare anche altre opere dello scrittore:

ci darete animo di darvi l'Ercole del medesimo auttore condotto a grato e dilettevole fine, e insieme un buon numero di tragedie, di felici e d'infelici soccessi, con varie sorti di rime, in diverse materie, leggiadramente composte. $^{17}$

Il progetto restò disatteso, perché Giraldi non portò a termine la redazione definitiva dell'Ercole ${ }^{18}$ e non consegnò mai per la stampa le sue tragedie inedite.

Quanto osservato finora induce dunque a ribadire che le tragedie giraldiane non avevano, nelle intenzioni dell'autore, una esclusiva destinazione scenica e riscuotevano ai suoi occhi un credito sufficiente per meritare le cure e le attenzioni che, di fatto, Giraldi dedicò loro, pur senza giungere all'approdo della divulgazione tipografica.

La mia ipotesi è che Giraldi si era imposto una meta ambiziosa, sul versante della teoria e della prassi drammaturgica, che non fece in tempo a raggiungere compiutamente, non tanto per la problematicità del tentativo di ricondurre ai canoni classici tutto quel complesso di innovazioni via via programmaticamente apportate ai generi teatrali, ma soprattutto, dopo l'Orbecche e le tragedie storiche, per l'insoddisfazione verso un impianto tradizionale, imperniato sui temi della vendetta e del suicidio, in contrasto con l'esigenza di promuovere più incisivamente $i$ valori cristiani fondati sulla fede e sul perdono; ${ }^{19}$ insoddisfazione ideologica da cui scaturi l'elaborazione della tragedia a lieto fine, che, giudicata 'inferiore' da Aristotele, scardinava i parametri consolidati del genere e apriva la strada a molteplici soluzioni tematiche e strutturali, meritevoli di altrettante giustificazioni teoriche. ${ }^{20}$

Fin dallinizio della propria carriera drammaturgica Giraldi aveva affrontato, con notevole consapevolezza critica, il problema spinoso della conciliazione tra l'osservanza del dettato aristotelico e l'adozione di schemi nuovi, che rispondessero alle esigenze e ai gusti moderni.

Il primo impianto del Discorso intorno al comporre delle comedie e delle tragedie risale al $1543{ }^{21}$ prima che uscissero $i$ 
commenti di Francesco Robortello e di Vincenzo Maggi ${ }^{22}$ alla Poetica aristotelica e dopo che Giraldi aveva composto le prime sue tragedie (l'Orbecche, la Didone, la Cleopatra, l'Altile). L'impegno era contestualmente quello di un'interpretazione corretta della Poetica di Aristotele e dell'elaborazione di una grammatica tragica efficace e coerente, ma soprattutto della legittimazione di tutti quegli aspetti drammaturgici che erano apparsi contrari ai precetti aristotelici. Già con la lettera a Ercole II d'Este, scritta nel Is4I per confutare le critiche mosse alla sua Didone ${ }^{23}$ (pubblicamente letta «alla presenza di tanti begli ingegni e nobili spiriti»), ${ }^{24}$ Giraldi si era soffermato sulla liceità delle innovazioni, facendo leva non solo sul principio di una normale evoluzione storica delle forme poetiche, ma anche sull'assen za di una dimensione rigidamente precettistica della Poetica aristotelica:

E se forse in qualche parte mi son partito dalle regole che dà Aristotile per conformarmi co' costumi de' tempi nostri, l'ho io fatto coll'essempio degli antichi, perché si vede che altrimente diede il principio alle sue favole Euripide che Sofocle, e con altro modo disposero le loro favole i Romani, come poco ha dicemo, che i Greci. Ed oltre a ciò, lo mi ha concesso il medesimo Aristotele, il quale non vieta punto, quando ciò richiede o luogo, o tempo, o la qualità delle cose che sono in maneggio, il partirci alquanto da quell'arte ch'egli ha ridotta sotto i precetti che dati ci ha. ${ }^{25}$

L'affermazione implica il progetto di rivisitazione dei generi teatrali, che era il frutto di una riflessione sulle tecniche della drammaturgia antica e, insieme, sulla materia e le forme più idonee al teatro moderno. Era forte, d'altra parte, la consapevolezza dell'autore di muoversi in un terreno accidentato, sia per il fatto che il genere della tragedia era stato in tempi moderni fino a quel momento trascurato, e sia per le difficoltà interpretative connesse alla Poetica aristotelica, imputabili non soltanto all'assenza, agli inizi degli anni Quaranta, di adeguati interventi esegetici, ma anche alla concreta circostanza della perdita di molti di quei testi teatrali sul cui esempio Aristotele stesso aveva elaborato la definizione della tragedia. Tali preoccupazioni, al momento della prima uscita a stampa dell'Orbecche (Venezia, Aldo, I543) erano cosi espresse da Giraldi nella famosa dedica a Ercole II premessa alla tragedia: ${ }^{26}$ 
Dura cosa è, illustrissimo Signore, a scrittori di qualunque sorte fuggire a questi tempi i morsi dell'invidia [...]. E posto che ciò sia difficile in ogni sorte di composizione, egli è sommamente difficile quando altri si dà a scrivere in quella maniera de' poemi che sono stati per tanti secoli tralasciati, ch'appena di loro vi resta una breve ombra. Di qui è ch'io istimo che sia quasi impossibile che coloro i morsi dell'invidia fuggano i quali si danno a comporre nuove tragedie a questi tempi, l'uso delle quali, solo maestro di tutte le cose, per la gran lascivia del mondo, com'io credo, è in tutto mancato, e appresso e Greci, che la tragedia trovaro, e appresso e Latini, che, togliendola da essi, senza alcun dubbio, assai più grave la fecero. Ed anchora ch'Aristotile ci dia il modo di comporle, egli, oltre la sua natìa oscuritade, la quale (come sapete) è somma, riman tanto oscuro e pieno di tante tenebre per non essere gli auttori de' quali egli adduce l'auttoritadi e gli essempi per confirmazione degli ordini e delle leggi ch'egli impone agli scrittori d'esse, ch'a ffatica è intesa non dirò l'arte ch'egli insegna, ma la diffinizione ch'egli dà della tragedia.

Nel I543 Giraldi aveva già pertanto enucleato alcuni fondamentali nodi, difficili da districare, della scrittura drammaturgica, dopo averne sperimentato le principali opzioni alternative: le tragedie ispirate a un soggetto noto (Didone $e$ Cleopatra) e le tragedie di nuova invenzione (Orbecche $e$ Altile), quelle con finale tragico (le prime tre), quelle con epilogo felice (l'ultima). Soprattutto l'oscurità' della Poetica si poneva all'origine delle divergenze degli scrittori sulla concezione della tragedia: l'ambiguità e la laconicità di alcune affermazioni contenute nella Poetica, tuttora oggetto di discussione critico-filologica, favorivano lo sviluppo di posizioni contrastanti, tutte allo stesso modo sostenute dalla medesima auctoritas aristotelica. Risultava non univoca persino l'interpretazione del passo relativo alla definizione della tragedia e del principio della catarsi (Poetica I449b 24-28):

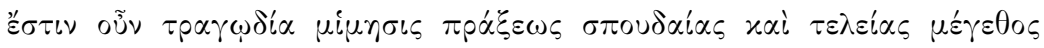

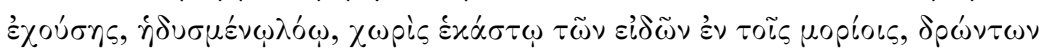

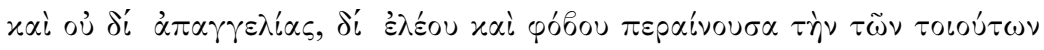

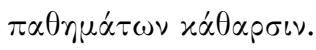

Il passo risulta variamente tradotto, anche nelle edizioni moderne, ${ }^{27}$ e il divario interpretativo riguarda soprattutto il tema della catarsi, in quanto pietà e paura possono essere intese o come strumenti per tenere 
lontane le passioni nocive, o come sentimenti negativi da esorcizzare. La traduzione di Alessandro de' Pazzi ("Tragoedia [...] per misericordiam vero atque terrorem pertubationes huiusmodi purgans») ${ }^{28}$ poteva orientare verso la seconda soluzione, accolta da Francesco Robortello, che identificava, appunto, le «perturbationes huiusmodi» proprio con la pietà e la paura. ${ }^{29}$ Assistendo alle rappresentazioni tragiche gli spettatori (o gli uditori) partecipano infatti emotivamente alle azioni rappresentate, imparando a vincere la pietà e la paura e a sostenere con maggiore forza e coraggio quelle stesse avversità subite dai protagonisti tragici. Non altrettanto convinto di questa interpretazione è Vincenzo Maggi, il quale, commentando il medesimo passo della Poetica, ${ }^{3 \circ}$ esclude che secondo Aristotele le passioni da purgare possano essere il terrore e la misericordia, ${ }^{3 \mathrm{I}}$ le quali costituiscono invece il fondamentale deterrente per le azioni delittuose. Giraldi si attiene a tale linea esegetica quando scrive che

la tragedia, o sia di fin lieto o d'infelice, col miserabile et col terribile purga gli animi da' vitii et gl'induce a' buoni costumi. ${ }^{32}$

$\dot{E}$ da notare che tale formulazione, rispetto alla definizione aristotelica, introduce due essenziali precisazioni tendenti a rendere canonico anche il lieto fine e a sottolineare le funzioni pedagogiche della tragedia, togliendo ogni ambiguità al concetto di catarsi, intesa quale purificazione dai vizi mediante $i$ sentimenti di pietà e terrore. Il meccanismo catartico è, peraltro, minutamente illustrato, ponendo la commiserazione e la paura come effetto della valutazione di una sproporzione tra l'errore involontario commesso dal protagonista dell'azione tragica e il supplizio subito. ${ }^{33}$ Lo spettatore prende atto delle conseguenze nefaste di una colpa involontaria e impara ad astenersi da quelle azioni per non incorrere nella medesima pena o in pene ancora più gravi:

Perché lo spettatore, con tacita consequenza, seco dice: «se questi, per errore commesso non volontariamente, tanto male ha sofferto quanto vedo io hora, che sarebbe di me se forse volontariamente commettessi questo peccato?». ${ }^{34}$

Tale meccanismo, tuttavia, perfettamente consono alle tragedie con epilogo tragico e con contenuti violenti e orrorosi, non appare attuabile allo stesso modo nelle tragedie a lieto fine, nelle quali solo $i$ personaggi 
malvagi subiscono le pene di cui sono degni, mentre i protagonisti si riscattano alla fine dai loro peccati veniali approdando ad un esito positivo delle vicende. Giraldi avvertì questa carenza programmatica e si impegnò a colmarla con alcune aggiunte manoscritte alla sua copia di lavoro dei Discorsi. Una di queste aggiunte è contigua alla riflessione sopra citata:

$<$ Può $>$ ancor purgar gli ani $<$ mi $>$ la favola tragica $\mathrm{da}<$ lle per $>$ turbationi che nasco $<$ no $>$ dalla infelicità de' casi $<$ hum $>$ ani, perché le miserie $<\mathrm{q}>$ ui spesse volte apport $<$ ano $>$ nelle aversità consola $<$ tion $>$ e agli afflitti. La onde, $<$ sen $>$ tendo gli spettatori, o co $<$ loro $>$ che leggono, quali et $<$ quante $>$ siano le passioni de $<$ gli huomini $>$ et le revolutioni de' $<$ f $>$ ati loro, prendono al $<$ quanto $>$ di quiete nelle tur $<$ batio $>$ ni loro, conformandosi $<$ alla $>$ necessità, parendo $\mathrm{lo}<$ ro $\mathrm{c}>$ he non sia loro avenu $<$ ta $\mathrm{co}>$ sa fuori della conditio $<$ ne $d>$ ella natura humana. $<\mathrm{La}$ qua $>$ le naturalmente è sotto $<$ posta $>$ a tutti i colpi della $<$ fort $>$ una. ${ }^{35}$

La tragedia, dunque, consente allo spettatore o al lettore anche di prendere coscienza della precarietà della condizione umana e di affrontare con la forza necessaria le avversità che comunemente tormentano gli uomini. Con tale osservazione Giraldi si avvicina all' interpretazione di Robortello, sopra ricordata, che attribuisce alla catarsi una funzione consolatoria, di liberazione da paure e turbamenti. Ma questa formulazione è immediatamente dopo compensata dalla dichiarazione di preferenza per la tipologia di catarsi descritta prima, ${ }^{36}$ quella cioè atta a purificare dai vizi. E questo appare un nodo cruciale della speculazione giraldiana, perché, se nel confronto tra queste due tipologie di catarsi egli privilegia sotto il profilo teorico la prima, è la seconda poi a prendere il sopravvento nella sua prassi drammaturgica, con l'elaborazione di testi tragici a lieto fine (come le tragedie Altile, Antivalomeni, Arrenopia, Epizia, Eufimia, Selene), i cui protagonisti, per errori dovuti a inesperienza e ingenuità e per la malvagità altrui, subiscono tutta una serie di eventi avversi prima di approdare a una soluzione positiva delle loro vicende.

Un limite all'esplicazione di una funzione catartica 'consolatoria' è dato comunque dal fatto che i protagonisti tragici debbano essere 'mezzani' (cioè debbano aver commesso qualche colpa involontaria per ignoranza e non per malizia): dal processo catartico si dichiarano pertanto esclusi tutti quegli spettatori che hanno provato sofferenze senza aver 
commesso alcuna colpa e che non possono trarre consolazione immedesimandosi in vicende nelle quali le avversità appaiano come conseguenze di errori, sia pure veniali. ${ }^{37}$ Come bo dimostrato in altra sede, ${ }^{38}$ Giraldi non deroga in alcun modo al principio della 'mezzanità' dei personaggi, ma fa leva sull'analisi introspettiva dei caratteri e su una costruzione della fabula idonea a far emergere $i$ rapporti di causaeffetto delle vicende rappresentate, secondo una dimensione nella quale deve risaltare il grado di responsabilità personale nel rivolgimento dei casi umani e il concetto di 'colpa' va commisurato ai presupposti e alle finalità delle azioni e alle caratteristiche dei personaggi, in base a regole di decoro e verosimiglianza. ${ }^{39}$ La riflessione sulle dinamiche catartiche della tragedia a lieto fine costitui un momento caratterizzante della revisione dei Discorsi: essa riguarda alcune aggiunte autografe volte a dimostrare anzitutto come questo tipo di tragedia sia avallata da Aristotele $^{4 \circ}$ e abbia avuto tra $i$ suoi cultori Sofocle, nell'Elettra, ed Euripide nell'Ifigenia in Aulide e nell'Ifigenia taurica, nell'Alcesti, nello Ione e nell'Elena. ${ }^{4 \mathrm{I}}$ La scelta del lieto fine è presentata in prima istanza, nella redazione a stampa, come una scelta necessaria a soddisfare le esigenze degli spettatori, ${ }^{22}$ e anzi, Giraldi rileva la distinzione, entro la categoria dei fruitori del teatro, tra lettori e spettatori, $i$ primi dei quali avvezzi a una tradizionale strutttura della fabula, con epilogo infelice o terrificante, $i$ secondi propensi ad assistere a spettacoli più rassicuranti. ${ }^{43}$ Se, dunque, la tragedia a lieto fine è concepita esclusivamente per la rappresentazione e non anche per la lettura, ciò potrebbe costituire una motivazione plausibile della fortuna editoriale dell'Orbecche e della mancata edizione delle altre tragedie a epilogo felice, ma non vi sarebbe comunque una spiegazione soddisfacente per la mancata edizione della Didone e della Cleopatra, che, in quanto tragedie storiche con epilogo infelice, avrebbero potuto essere destinate anche a un pubblico di lettori. La frattura, in certo modo esterna e strumentale, tra $i$ due tipi di fruizione (lettura e spettacolo), viene peraltro sanata (anche mediante l'innesto di integrazioni autografe), dalla ricerca giraldiana di più cogenti ragioni ideologiche e retoriche. Giraldi insiste soprattutto sul fatto che le affinità tra commedia e tragedia a lieto fine siano soltanto apparenti, dato che questo genere di tragedia rappresenta la morte di coloro che hanno causato ingiustamente le sofferenze dei protagonisti dell'azione, ${ }^{44}$ e che, contrariamente all'opinione comune, pietà e commiserazione siano ingredienti fondamentali 
anche della tragedia che si conclude felicemente. ${ }^{45}$ Inoltre, la caratterizzazione tragica si esplica non soltanto attraverso le azioni, ma anche attraverso tutti gli elementi atti a qualificare $i$ personaggi e le loro dinamiche comportamentali. Giraldi riassume questi fondamentali ingredienti del teatro (sia esso comico o tragico) sotto le etichette di 'sentenza' e 'costume', dove con 'sentenza' fa riferimento a quelle "perturbationi dell' animo» che sono causa della peripezia, ${ }^{46}$ e per 'costume' intende, filosoficamente, l'abito (l'vं$\theta \circ \varsigma$ aristotelico), ovvero l'attitudine dei personaggi al vizio o alla virtù. ${ }^{47}$ La fabula ha una efficacia etico-pedagogica solo se le azioni rappresentate sono accompagnate e sostenute da idonee forme espressive, ${ }^{48}$ da un'attenzione al decoro, alla verosimiglianza e alla coerenza nella presentazione di fatti e personaggi $^{49}$ e alle caratteristiche del linguaggio dei protagonisti ${ }^{\circ 0}$ o del messaggero, deputato a riferire nelle tragedie le azioni cruente non rappresentabili in scena. ${ }^{5 \mathrm{I}}$ Tuttavia la tragedia è preposta ad accogliere contenuti più 'alti' e «il parlare della tragedia vuole esser grande, reale et magnifico, et figurato, quello della comedia, semplice, puro, famigliare et convenevole alle persone del popolo».52 Questo è il fondamentale discrimine (oltre all'ambientazione, regale in un caso, popolaresca nell'altro) che Giraldi individua tra i due generi, facendo si che, sul piano teorico e programmatico, la tragedia a lieto fine sia assimilabile non alla commedia, ma alla tragedia con epilogo infelice per tecniche drammaturgiche e aspetti formali.

Va tuttavia ricordato come in alcune tragedie giraldiane le peculiarità distintive rispetto alla commedia tendano a sfumare: in particolare negli Antivalomeni e nell'Epizia. La tragedia Antivalomeni, la cui composizione è precedente al luglio del I548 (data della sua prima rappresentazione), condivide, oltre al lieto fine, una serie di caratteristiche con la commedia Eudemoni, a partire dal titolo (non riferito a un nome proprio di persona) ${ }^{53}$ e dallintreccio amoroso tipico della commedia classica, sul genere degli Adelphoi di Terenzio. ${ }^{54}$ L'Epizia (elaborata negli ultimi anni del periodo ferrarese di Giraldi, o al più tardi nei primi anni del soggiorno piemontese) ba persino un'ambientazione non regale, perché la protagonista è una «vergine illustre», non una principessa o una regina. ${ }^{5}$ In entrambe le fabulae nessun malvagio muore, ma prevale la logica del pentimento e del perdono. Queste innovazioni si iscrivono in un itinerario sperimentale coerente e sempre più intensamente intrecciato a istanze etico-pedagogiche, ma, se la prima 
tragedia (Orbecche) è approdata alle stampe e le altre successive, che dovrebbero considerarsi il frutto di un'esperienza drammaturgica più consapevole e matura, sono rimaste inedite, cio rende legittima la seguente riflessione: Giraldi non poteva approvare la pubblicazione dei propri successivi testi teatrali senza aver affrontato complessivamente tutte le questioni teoriche e ideologiche sottese alla prassi drammaturgica. I dati in nostro possesso dimostrano che si trattò di un lavoro perennemente in fieri, di un vicendevole e progressivo affinarsi di assunti teorici e sperimentazione teatrale, quando innovazioni sempre piu ardite richiedevano una giustificazione teoretica e quando la regola inderogabile del «decoro» e del «convenevole» imponeva una rivisitazione delle tematiche e delle strutture tragiche, in sintonia con nuove esigenze socio-politiche e culturali; un lavoro sempre sostenuto dalla convinzione della validità della strada (sia pure impervia e piena di rischi) intrapresa. ${ }^{56}$ La produzione giraldiana (nel caso specifico la drammaturgia, ma anche l'epica) va studiata abbandonando l'errato presupposto che l'autore sia pervenuto all'approdo definitivo del lavoro redazionale: $i$ Discorsi, stampati nel I554, non attestano una 'definitiva' volontà dell'autore, mentre l'esemplare ferrarese con correzioni e aggiunte autografe offre testimonianza di un lavoro di revisione non portato a termine, interrotto e abbandonato quando Giraldi, ormai stanco e malato, dedicava le sue ultime energie all' insegnamento di retorica presso l'Università di Torino e poi presso l'Università di Pavia. ${ }^{57}$ La stampa postuma delle tragedie, d'altra parte, con tutti $i$ suoi errori tipografici (ma talora anche problemi metrici e lacune testuali), rende probabile l'ipotesi che Celso Giraldi abbia consegnato in tipografia un manoscritto paterno estremamente tormentato, con stesure dei testi tragici (se si eccettua l'Orbecche) non pervenute ad un ultimo lavoro di lima. La perdita dell'intero corpus di manoscritti delle tragedie ${ }^{8}$ non consente, purtroppo, di verificare la tipologia redazionale e la consistenza e qualità dei presunti interventi d'autore sui testi tragici. Indizi significativi di incompintezza, di varianti alternative o di stratificazioni redazionali potranno solo eventualmente essere rintracciati sulla scorta di una rinnovata ricognizione filologica di tutte le tragedie. ${ }^{59}$ A conforto di questa ipotesi vi è anche la tipologia di stratificazioni redazionali che caratterizza l'esemplare con correzioni autografe dei Discorsi (Cl. I 9o), l'autografo con i canti dell'Ercole (Cl. I 406), l'autografo della commedia Eudemoni (Cl. I 407), tutti conservati all'Ariostea di 
Ferrara. ${ }^{60}$ D'altra parte, a causa della mancata conoscenza della fisionomia originaria delle tragedie giraldiane (fatta eccezione per l'Orbecche, pubblicata in tempi molto vicini a quelli della sua elaborazione e rappresentazione scenica), la prospettiva critica risulta spesso falsata, anche per la difficoltà di una corretta valutazione del rapporto tra $i$ testi tragici e le novelle degli Ecatommiti, che, come afferma Giraldi stesso, ne hanno costituito la fonte. Infatti i rapporti tra novelle e tragedie non devono essere considerati lineari e univoci, perché la revisione delle novelle (di cui pure manca attestazione autografa, ma che presumibilmente avvenne in larga misura a ridosso dell'edizione del I565) tiene conto dell'esperienza della sperimentazione tragica, cosi come quest'ultima si appoggia e si perfeziona in concomitanza con le riflessioni teoriche dei Discorsi. ${ }^{61}$ Accanto all'esemplare ferrarese dei Discorsi $e$ allo scartafaccio delle tragedie, sullo scrittoio di Giraldi doveva esserci, in uno stesso lasso di tempo, anche il corposo manoscritto degli Ecatommiti, soggetto fino all'ultimo a modifiche e ripensamenti, ${ }^{62}$ nell'ottica di un costante confronto tra sistemi mimetici e narrativi ugualmente finalizzati a una strategia edificante. ${ }^{63}$

Occorrerà prendere atto (traendone le necessarie conseguenze criticofilologiche), che Celso Giraldi, ritenendo di onorare la memoria del padre, divulgò ai posteri opere che non erano pervenute a uno stadio di compintezza formale e non avevano ricevuto l'imprimatur da parte dell'autore.

Susanna Villari 
I. C. Dionisotti, recensione a P. R. Horne, The tragedies of Giambattista Cinthio Giraldi, Oxford, Oxford University Press, 1962, in «Giornale storico della letteratura italiana», I40 (I963), pp. I I 4-2 I.

2. Ibidem, pp. I I 5- I6.

3. Ibidem, p. I 16 .

4. In proposito, anche per la bibliografia relativa, cfr. almeno S. Jossa, Rappresentazione e scrittura. La crisi delle forme poetiche rinascimentali (I540-I560), Napoli, Vivarium, I996; V. Gallo, Da Trissino a Giraldi. Miti e topica tragica, Manziana (Roma), Vecchiarelli, 2005 .

5. Cfr. Jossa, Rappresentazione e scrittura cit., p. 23: «al realismo spettacolare del Giraldi, fondato sulla rappresentazione dell'“orrore", della morte e del sangue, lo Speroni contrappone il gioco verbale, la metafora, l'illusione scenica. [...] Da una parte, appunto, la rappresentazione, lo spettacolo, dall'altra la scrittura, il laboratorio formale».

6. Cfr. Giovan Battista Giraldi Cinthio, Orbecche, prologo, vv. 5-6 (cito dall'edizione a cura di R. Cremante, in Teatro del Cinquecento. Tomo I. La tragedia, MilanoNapoli, Ricciardi, pp. 26I-448, alle pp. 291-92).

7. Cfr. Giraldi Cinthio, Orbecche, «La tragedia a chi legge», vv. 317I-I99; vv. 3244-47; vv. 3259-6I (Teatro cit., pp. 433-48).

8. Per la storia compositiva degli Ecatommiti cfr. S. Villari, Introduzione a GIOvaN Battista Giraldi, Gli Ecatommiti, Roma, Salerno Ed., 20 i 2, soprattutto le pp. XXVI. Oltre all'Orbecche (tratta da Ecatom. II 2), sono costruite sulle trame di novelle giraldiane le tragedie Altile (Ecatom. II 3), Antivalomeni (Ecatom. II 9), Arrenopia (Ecatom. III I), Selene (Ecatom. V I), Eufimia (Ecatom. VIII ıо), Epizia (Ecatom. VIII 5).

9. «Le offro Epiz̨ia tragedia di mio padre per ancora né in publico esposta, né rappresentata in scena». Così scrive Celso Giraldi nella dedica a Margherita Gonzaga, duchessa di Ferrara, premessa all'Epizia, all'interno della raccolta di tragedie giraldiane curata dallo stesso Celso, figlio dell'autore (Le tragedie di M. Gio Battista Giraldi Cinthio nobile ferrarese, cioè Orbecche, Altile, Didone, Antivalomeni, Cleopatra, Arrenopia, Euphimia, Epitia, Selene, Venezia, Giulio Cesare Cagnacini, I 583 , 3 voll., ciascuna tragedia ha una sua paginazione autonoma). Sarà necessario destinare ad altra sede la necessaria illustrazione delle peculiarità bibliografiche della raccolta.

ı. Per una ricognizione dei dati relativi a circostanze e cronologia delle rappresentazioni delle tragedie giraldiane rinvio a Giovan Battista Giraldi Cinthio, Discorsi intorno al comporre rivisti dall'autore nell'esemplare ferrarese $\mathrm{Cl}$. I 90, a cura di S. Villari, Messina, Centro interdipartimentale di Studi umanistici, 2002, pp. XLII-XLIII, n. 3 .

I I. Cfr. M. Pieri, Mettere in scena la tragedia. Le prove di Giraldi, in «Schifanoia», I 2 (I99I, fasc. monografico sugli Atti delle giornate di studio dedicate a Giovan Battista 
Giraldi Cinzio, organizzate dall'Istituto di studi rinascimentali e dal Centro di Studi su Matteo Bandello e la cultura rinascimentale nei giorni 27-29 aprile 1989), pp. I 29-42, a p. I 29: «egli cioè scriveva le sue tragedie per rappresentarle e le metteva in scena via via che veniva componendole, come accade a pochissimi altri autori cinquecenteschi e a nessun altro tragico, escluso forse l'eccezionale Groto».

I 2. Cfr. la lettera dedicatoria a Ercole II d'Este premessa all'Orbecche, in Cremante, Teatro cit., pp. 283-85. Per questi aspetti e per tutta la bibliografia relativa: F. Doglio, Sulle prime rappresentazioni dell"Orbecche' e Giraldi "corago", in «Critica letteraria», I 59-60 (2013: numero monografico dedicato al Convegno su Giovan Battista Giraldi Cinthio hombre de corte, preceptista y creador, Universitat de València 8 al Io de Noviembre de 2012, Atti a cura di I. Romera Pintor), pp. 29I-307.

I 3. GIRALDI, dedica all'Orbecche, in Teatro cit., p. 285 : «come i preghi degli amici mi constrinsero a comporla, così anco le costoro continove dimande m'hanno sforzato a lasciarla uscire».

14. Ibidem, p. 285.

I 5 . Dove è esplicito il riferimento alle «lodi» del duca e degli altri illustri signori. Pubblicata nell'edizione Cagnacini delle Tragedie in calce alla Didone, questa apologia si legge anche in Giovan Battista Giraldi Cinzio, Carteggio, a cura di S. Villari, Messina, Sicania, 1996, lett. 23, pp. I53-71. Cfr. p. I53, n. I per la datazione (I54I e non I543, come erroneamente riportato nella stampa Cagnacini).

i6. Si vedano soprattutto (in Giraldi Cinzio, Carteggio, lett. 49-5 I, pp. 235-38), le missive ad Ercole II d'Este che accompagnano l'allestimento degli Antivalomeni, importanti documenti del ruolo di Giraldi come "corago", ovvero regista teatrale (cfr. Doglio, Sulle prime rappresentazioni cit., pp. 306-307).

17. Cfr. Giraldi, Gli Ecatommiti, ed. cit., vol. III, p. i84i. Arlenio Arnoldo, intellettuale di origini fiamminghe, era colui che, allinterno della tipografia di Leonardo Torrentino, a Mondovì, si occupava della revisione dei testi e della promozione editoriale.

I 8. Di cui restano, autografe, le stesure di alcuni canti inediti (non inclusi tra quelli presenti nell'edizione parziale, Dell'Hercole [...] canti 26, Modena, Gadaldini, I 557, che Giraldi aveva voluto divulgare solo allo scopo di premunirsi da eventuali plagi). Sulla vicenda cfr. C. Molinari, I canti dell'Ercole' secondo l'autografo Cl. I 406 della BCAFe: note sulla vicenda redazionale del poema giraldiano, in «Critica letteraria», I 59-160 (2013), pp. 503-32.

I9. Va detto, tuttavia, per inciso, che il motivo della clemenza e del perdono si affaccia prepotentemente fin dall'Orbecche e dalla Cleopatra (si veda, nell'Orbecche, atto III scena II, il dialogo tra Malecche e Sulmone, e nella Cleopatra, atto II scena $\mathrm{V}$, il dialogo tra Ottavio e Mecenate); e la riflessione critica sul tema del suicidio attraversa pure la speculazione giraldiana dalle prime tragedie fino agli Ecatommiti, con una oggettiva valutazione che tiene conto, sul piano teoretico, dei più alti approdi, non sempre convergenti, della filosofia classica, e su un piano pragma- 
tico, delle concrete situazioni nelle quali il 'togliersi la vita' può avere, in casi estremi, attenuanti o giustificazioni. In proposito cfr. C. Lucas Fiorato, Giraldi Cinz̧io e il suicidio negli 'Ecatommiti', in «Critica letteraria», I 59-60 (201 3), pp. 443-59.

20. Ł̀ da tener presente in proposito l'osservazione di M. Pieri circa la passione del pubblico colto ferrarese per le questioni poetiche, che spiega la costante attenzione giraldiana alle implicazioni teoriche delle proprie sperimentazioni (Pieri, Mettere in scena cit., p. I 3 I).

2 I. Il Discorso intorno al comporre delle comedie et delle tragedie, scritto in forma epistolare ed indirizzato a Giulio Ponzio Ponzoni, allievo di Giraldi e attore, reca infatti la data del 20 aprile I 543 (cfr. GIRALDI, Discorsi, II 264; seguo la paragrafatura dell'edizione sopra citata).

22. Francisci Roborteldi Vtinensis In librum Aristotelis De arte poetica explicationes, Florentiae, Laurentius Torrentinus, i 548; Vincenti Madi Brixiani et Bartholomaei Lombardi Veronensis In Aristotelis librum de poetica communes explanationes, Venetiis, in officina Erasmiana Vincentii Valgrisii, I 50.

23. Giraldi Cinzio, Carteggio, lett. 23, pp. i 53 -7 I.

24. Ibidem, p. I 54.

25. Ibidem, p. I69.

26. Giraldi, Orbecche, in Teatro cit., p. 283.

27. Ad es. C. Gallavotti, in Aristotele, Dell'arte poetica, Milano, Mondadori (Fondazione Lorenzo Valla), I974, p. I9: «Tragedia è opera imitativa di un'azione seria, completa, con una certa estensione; eseguita con un linguaggio adorno distintamente nelle sue parti per ciascuna delle forme che impiega; condotta da personaggi in azione, e non esposta in maniera narrativa; adatta a suscitare pietà e paura, producendo di tali sentimenti la purificazione che i patimenti rappresentati comportano». Oppure M. Zanatta, in Aristotele, Retorica e Poetica, Torino, Utet, 2006, p. 600: «tragedia è mimesi di un'azione elevata e compiuta in se stessa, dotata di grandezza, con un linguaggio che dà piacere con ciascuna specie $<$ di abbellimenti> separatamente nelle parti, di persone che agiscono e non con una narrazione, la quale, tramite pietà e terrore, porta a compimento la purificazione delle passioni proprie di questo genere $<$ di azioni $>»$. Per la trasposizione italiana del termine x́á $0 \alpha \rho \sigma \nu$ si veda inoltre R. Tesi, Aristotele in italiano. I grecismi nelle traduzioni italiane della 'Poetica', Firenze, Accademia della Crusca, I997, pp. I66-67.

28. 'Purgando la tragedia, per mezzo della misericordia e del terrore, dai turbamenti di tal genere' (trad. mia); il testo latino di Alessandro de' Pazzi (Aristotelis Poetica [...] in Latinum conversa, Venetiis I 536) confluisce nelle particulae dei commenti di Robortello e Maggi, da cui attingo le citazioni.

29. Robortello, Explicationes, pp. 52-54.

30. Maggi, Explanationes, pp. 97-98. 
31. Ibidem, p. 98: «dubito Aristotelem nolle Tragoediae finem esse animam humanam a terrore misericordiave expurgare, sed his uti ad alias perturbationes ab animo removendas; ex quarum remotione animus virtutibus exornatur. Nam ira, verbi gratia, depulsa, succedit mansuetudo, expulsa avaritia, inducitur liberalitas, atque ita de ceteris» ('non credo che Aristotele volesse che il fine della tragedia consistesse nel purificare l'animo umano dal terrore e dalla misericordia, piuttosto credo che egli volesse servirsi di questi sentimenti per rimuovere dall'animo altre passioni; rimozione che comporta che l'animo si adorni di virtù. Infatti, ad esempio, rimossa l'ira, subentra la mansuetudine, rimossa l'avarizia, subentra la liberalità, e così via'; trad. mia).

\section{Giraldi, Discorsi, II i6.}

33. Ibidem, II 50: «pare allo spettatore che ad ogni modo fosse degna di qualche pena la persona che soffre il male, ma non già di così grave». Va ricordato che presupposto indispensabile per consentire tale immedesimazione è l'appartenenza del protagonista tragico alla categoria di persone «mezzane», ovvero né del tutto buone, né del tutto scellerate (cfr. II 50). Sulla questione: S. Villari, Giraldi e la teoria del personaggio 'mezzano'tra teatro e novellistica, in «Critica letteraria», I 59-I60 (2013), pp. 402-25.

34. Giraldi, Discorsi, II 53. Con un'integrazione autografa, poi cancellata, Giraldi concludeva il ragionamento sottolineando la rilevanza etico-civile del processo catartico: «et se astiene dal male operare» (che a sua volta sostituiva una precedente formulazione: «et questo pensiero il fa astenere dagli errori»). Cfr. l'esemplare ferrarese dei Discorsi, Ferrara, Bibl. Comun. Ariostea, Cl. I 90, a p. 2 I 8 (Ibidem, II 53). E ancora in un'altra aggiunta a p. 226 (Ibidem, II 93): «veggen < do $\mathrm{g}>$ li huomini di minore $<$ et $\mathrm{pi}>\mathrm{u}$ bassa conditione $\mathrm{i}$ tra $<$ vagli $>$, i pericoli et le afflittio $<$ ni $\mathrm{c}>$ he intervengono a perso $<$ ne di $>$ alto grado, argomenta $<$ no d $>$ al maggiore al minore, $<$ dicen $>$ do: se $<$ perso $>$ na tale $<$ incorr $>$ e in simili travagli et $<$ in $\mathrm{s}>$ imili calamità, che sarà $<$ di $\mathrm{n}>\mathrm{oi}$ di molto minore con $<$ ditio $>$ ne se forse in similli $<$ erro $>$ ri ci lascieremo condurre $<$ dal $>$ folle et disordinato ap $<$ peti $>$ to? Et da uno interno rimor $<$ dime $>$ nto tocchi, si riguardano $<$ da c $>$ osì fatti errori et anche $<$ dag $>$ li altri maggiori». Tra parentesi uncinate si pongono $i$ segmenti testuali non visibili nell'esemplare (perché vergati sui margini rifilati in seguito a una legatura settecentesca) e restaurati per congettura.

35. Aggiunta autografa, a p. 2 I 8 dell'esemplare citato (Ibidem, II 54).

36. Nella stessa aggiunta autografa: «Ma, quantunque <questa $>$ opinione paia assai $<$ conve $>$ nevole, a me nondimeno $<\mathrm{p}>$ iace più la prima» (Ibidem, II 5 ) ).

37. Ibidem, p. 232 (aggiunta manoscritta): «non reca ciò consolatione a quelli, che senza haver commesso <pecc>ato sono incorsi nelle tribulationi, come alla giornata ne veggiamo spessissime volte avenire».

38. Villari, Giraldi e la teoria cit. 
39. Giraldi, Discorsi, II 56: «Et nello eleggersi o formarsi queste attioni illustri, così dette non perché siano lodevoli o virtuose, ma perché vengono da grandissimi personaggi, non è se non bene haverle tali, quali le ricercano verisimilmente i tempi ne' quali scrive il poeta, quanto a' ragionamenti, a' costumi, al decoro et alle altre circonstanze della persona».

40. Cfr. Ibidem, II I 3 I-32, dove Giraldi, integrando la definizione aristotelica di «peripezia» quale «mutatione di fortuna felice alla infelice e da questa alla contraria» [cfr. Aristotele, Poetica, I452a, 22-23], sottolinea in un'aggiunta autografa che ciò «aviene quando $<\mathrm{la}$ co $>$ sa è condotta a mal fine da lieto $<\mathrm{co}>$ minciamento o $\mathrm{v}<$ ero $>$ a migl $<$ ior fine $>$ che non si pensava, et $<$ se bene $>$ Aristotile dia l'essemp $<$ io di $>$ ciò ne' casi infelici, $\mathrm{p}<$ are $>$ nondimeno che signifi $<$ chi $>$ questa voce mutatione $\mathrm{d}<$ ell'una $>$ et dell'altra fortuna, $<$ et che $>$ per essa signifi $<$ chi la $>$ mutatione dell'una $<$ et del $>$ '’altra tragedia, sì $\mathrm{c}<$ he le $>$ felici divengano $\mathrm{mi}<$ sere et le $>$ misere felici». Nonostante, dunque, Aristotele attribuisca maggiore autorità al modello di tragedia con conclusione infelice, il fatto stesso che egli contempli la peripezia come passaggio da uno stato infelice a uno felice conferma, secondo Giraldi, la liceità di entrambi i generi di tragedia.

\section{I. Ibidem, II, I 32 (aggiunta autografa).}

42. Ibidem, II, 65-66: «Et anchora che Seneca tra' Latini non habbia mai posta mano alle tragedie di fin felice, ma solo si sia dato alle meste [...], nondimeno noi, $\leq \mathrm{ad}>$ essempio di Sopho $<$ cle $>$ et di Euripide, n'habbiam composta alcuna a questa imagine, come l'Altile, la Selene, gli Antivalomeni et le altre, solo per servire agli spettatori et farle riuscire più grate in scena, et conformarmi più con l'uso de' nostri tempi. Che anchora che Aristotile dica che ciò è servire alla ignoranza degli spettatori [ARistotele, Poetica, I 453 a, 34-35], havendo però l'altra parte i difensori suoi, ho tenuto meglio sodisfare a chi ha ad ascoltare, con qualche minore eccellenza (quando fosse accettata per la migliore l'opinione di Aristotile), che con un poco più di grandezza dispiacere a coloro per piacere de' quali la favola si conduce in scena; che poco gioverebbe compor favola un poco più lodevole, et che poi ella si havesse a rappresentare odiosamente». La sezione evidenziata con sottolineatura è una significativa aggiunta marginale rispetto alla redazione a stampa: Giraldi si impegna a ricondurre le proprie scelte drammaturgiche entro il solco di una tradizione classica.

43. Cfr. Ibidem, II 67: «quelle [le tragedie] terribili (se gli animi degli spettatori forse le abhorriscono) possono essere delle scritture, queste di fin lieto delle rappresentationi».

\section{Ibidem, II 67 (aggiunta autografa).}

45. Cfr. Ibidem, II 90 (aggiunta autografa): «p $<$ rima $>$ che giunga l'attione al fine, avengono casi tanto terribili et compassionevoli, che gli spettatori non possono $\mathrm{c}<$ ontenere $>$ le lagrime per la pietà». Ma anche nella redazione a stampa questo aspetto risulta già opportunamente evidenziato (cfr. II 68: «Si debbono nondimeno far nascere gli avenimenti di queste men fiere tragedie in guisa che gli spettatori tra l'horrore et la compassione stiano sospesi insino al fine, il qual poscia, riuscendo allegro, gli lasci tutti consolati»), con insistenza sullo stato di suspense nel quale devono essere tenuti fino all'ultimo gli uditori. 
46. Cfr. Ibidem, II I 46.

47. Cfr. Ibidem, II I 48 .

48. Cfr. Ibidem, II i 47: «Ma anchora che le parole et le attioni siano inditii della voluntà, non si dee però pensare che nel mostrare la intentione altrui, sì ch'ella sia atta alla scena, le attioni sole bastino, perché, quantunque uno uccidesse un altro, atto all'horrore e alla compassione, non verrebbe indi né questa né quello, se non vi fosse chi con acconcie parole commovesse gli animi degli spettatori. Per la qual cosa si può vedere che il parlare è così necessario alla sentenza, che ella senza lui se ne rimarrebbe gelata, quantunque ella per l'attione si dimostrasse. Et però si può conchiudere per hora che la sentenza non è altro che l'altrui intentione, o voluntà che noi vogliam chiamare, espressa con parole acconcie a mover terrore e compassione nella tragedia et nella comedia ad indurre turbe popolaresche et travagli tra donne et huomini innamorati, tra servi et patroni, tra marito et moglie, $<$ et $\mathrm{pa}>$ dri et figliuoli et $<$ altr $>$ i simili, col mezzo di roffiani, di parasiti et di altre tali persone; per opra delle quali anco spesso si acquetano esse turbe e si riducono a pace et a quiete» (il segmento testuale sottolineato costituisce un'aggiunta autografa).

49. Cfr. Ibidem, II i 5 I, dove Giraldi porta ad esempio la fisionomia di un uomo coraggioso, le cui azioni dovranno essere, nel contesto della fabula tragica, coerenti con il suo profilo iniziale di uomo portato a difendersi dalle ingiurie affrontando apertamente e senza insidie i suoi antagonisti. Ma cfr. anche II I 97-202, sul decoro dei caratteri e sulla conformità dei costumi, e sugli aspetti indecorosi (e perciò non degni di imitazione) del teatro tragico greco e della commedia plautina.

50. Cfr. Ibidem, II 208: «egli è da porre quanta più si può diligenza che tale egli [il parlare] riesca nella scena quale si conviene alle qualità delle persone che vi s'introducono; et far sì ch'i giovani da giovani favellino, i vecchi da vecchi [...] havendo però sempre riguardo alla qualità e allo stato della persona. Perché altrimenti favellerà un giovane del popolo et altrimenti un giovane reale, et altra prudenza mostrerà un vecchio re, che un padre di famiglia».

51. Il messo deve esprimere tutta l'emozione suscitata dall'evento al quale ha assistito: «l'horrore del caso avenuto induce stupore e un certo raccapriccio, che fa uscire chi l'ha veduto come di sé, onde egli, quasi tocco da furore, non può mandar fuori se non parole grandi e piene dello spavento ch'egli ha nell'animo; et devesi egli estendere in narrare il caso miserabile et horribile, in mostrare gli atti, i pianti, le parole, la crudeltà, la desperatione, la maniera con la qual morto cadde il miserabile, et l'altre cose, le quali tutte si chiuderebbeno altrimenti con dire: 'et egli fu morto crudelissimamente'» (Ibidem, II 2 I 2). Il brano costituisce un'ulteriore esemplificazione del rapporto tra la rappresentazione di un'azione scenica e la forma espressiva (II I 47), "perché, quantunque uno uccidesse un altro, atto all'horrore et alla compassione, non verrebbe indi né questa né quello, se non vi fosse chi con acconce parole commovesse gli animi degli spettatori». E Giraldi dedica, peraltro, molte pagine del suo trattato per spiegare come, per essere incisivo, il linguaggio debba essere semplice, non viziato da tortuosi modi di dire, «acciò che lo splendor delle parole non offuschi la luce delle sentenze, et 
le faccia divenir meno pregiate et meno efficaci di quel che debbono essere» (II 224).

52. Ibidem, II 209.

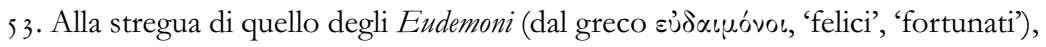
il titolo Antivalomeni sembra coniugato sul verbo greco $\alpha \nu \tau \iota \beta \dot{\alpha} \lambda \lambda \omega$ (letteralmente 'ribatto lanciando dardi'), con allusione al reciproco scambio di attacchi, di inganni e di ruoli tra i protagonisti della vicenda.

54. Cfr. I. Romera Pintor, Introduzione a Giambattista Giraldi Cinthio, Gli Antivalomeni, edición, introducción y notas de I. Romera Pintor, Madrid, Editorial Complutense, 2008, p. XXI.

55. Cfr. Giraldi, Epizia, argomento (Epizia. An italian Renaissance tragedy, by G.B. GiRALDI, edited with introduction, Notes and Glossary by P. Horne, Lewiston, Queeston, Lampeter, Edwin Mellen Press, I996, p. I). Per l'Epizia rinvio anche a C. Castorina, L'Epizia' di Giraldi Cinzio. Edizione critica' (tesi di laurea in Lettere - curr. moderno, anno acc. 20I 2-20I3, Dipartimento di Civiltà antiche e moderne, Università di Messina, rel. prof.ssa S. Villari).

56. Basti ricordare una delle postille ai Discorsi (non databile con precisione, ma certamente posteriore al I 554), con cui lo scrittore ferrarese rivendicava con orgoglio la propria scelta del lieto fine nelle tragedie, a dispetto di «coloro che non sanno porre il piede se non nelle vestigia altrui» e con l'auspicio dell'approvazione di «coloro che sono d'ingegno più vivace et conoscono che le cose si vanno tuttavia avanzando di età in età, et che molte usanze antiche se ne cadono et se ne vanno in oblivione, et molte alla giornata di novo rinascono, le quali, se portano seco il decoro col verisimile, non solamente non sono schifevoli, ma riescono grate nel cospetto de' giudiciosi» (GIRALDI, Discorsi, II 236, aggiunta autografa).

57. Si trasferì nel novembre del I 566 a Torino e alla fine del I 568 a Pavia (cfr. anche per i riferimenti bibliografici GIRALDI, Carteggio, pp. 4I9 n. e 422-23 ก. 2).

58. Probabilmente essi furono cestinati proprio dopo la realizzazione della stampa del i 583 , come era allora normale consuetudine nelle tipografie.

59. L'ipotesi che il manoscritto giraldiano della tragedia, entrato in tipografia, «non fosse pervenuto alla sua forma definitiva» è stata formulata, per l'Epiziıa, da C. Castorina, riflettendo su quei problemi testuali e metrici non genericamente classificabili come meri errori tipografici (Nota al testo premessa a Epiżia cit., p. 4).

6o. Per i Discorsi e l'Ercole rinvio agli studi citati sopra. Per gli Eudemoni cfr. A. Guidotti, Gli Eudemoni e le teorie giraldiane sulla commedia, in «Schifanoia», I 2 (I99I), pp. I 89-99. Cfr. inoltre l'edizione approntata da P. Horne (Gli Eudemoni. An Italian Renaissance Comedy, by G.B. GIRALDI, Lewiston, Queeston, Lampeter, Edwin Mellen Press, I 999), che tuttavia è priva di un apparato filologico che renda conto di correzioni e varianti d'autore. 
6r. Tempi e ragioni della rielaborazione autografa dei Discorsi sono ricostruiti in Villari, sezione introduttiva a GIRALDI, Discorsi, cit., pp. XLIX-CXXIII; cfr. in particolare a p. CXXXI, dove si sottolinea che il lavoro di lima condotto dall'autore sull'esemplare ferrarese «in più punti e per diversi aspetti risulta non perfettamente compiuto».

62. Una variante di scarto, attestata da alcuni esemplari della princeps degli Ecatommiti, si offre in particolare agli studiosi come clamoroso indizio della tipologia del lavoro di Giraldi condotto fino all'ultimo sul manoscritto (perduto) degli Ecatommiti. La variante riguarda la didascalia introduttiva della novella (VIII 7), che, nel suo 'stato' definitivo e corretto, è coerente con l'epilogo della novella, così come lo leggiamo in tutte le edizioni dell'opera (la nobile Semne, persuasa dai parenti, rinuncia a darsi morte); secondo la lezione di scarto della didascalia, Semne invece «si dà morte». Ciò è prova inequivocabile della circostanza che Giraldi aveva modificato, nel manoscritto destinato alla tipografia, l'epilogo della novella, dimenticandosi di correggere contestualmente anche il contenuto della didascalia. È apparsa questa l'unica spiegazione plausibile per la variante («si dà morte» / «si vuol dar morte, ma, impedita da' parenti, vive santamente»), che non può essere riconducibile a una svista tipografica poi sanata, ma presuppone una stratificazione compositiva, di cui offre una testimonianza indiretta la sopravvivenza di fascicoli con la lezione scartata. La modifica giraldiana dell'epilogo si può ricondurre, nel complesso, alla volontà di equilibrare i rapporti tra novelle a lieto fine e novelle con finale tragico, movimentando la casistica di situazioni presentate. Il fenomeno delle varianti di 'stato', oggetto di attenzione critica nel contesto delle metodologie della Textual bibliography, è comune alle procedure tipografiche all'epoca della stampa manuale: il controllo delle bozze avveniva spesso mentre la tiratura era già avviata e la segnalazione di errori da correggere avveniva quando già alcuni fogli di stampa erano già passati sotto il torchio. Dopo la ripresa della tiratura, in seguito all'intervento di correzione sulla forma di stampa, i fogli contenenti la lezione scorretta sopravvivevano accanto ai nuovi fogli con la lezione definitiva (da qui deriva la difformità degli esemplari, destinata ad emergere solo attraverso minuziose collazioni): sul tema cfr. almeno C. Fahy, Edizione, impressione, emissione, stato, in Saggi di bibliografia testuale, Padova, Antenore, I988, pp. 65-88. Per il caso specifico delle varianti interne alla princeps degli Ecatommiti, cfr. invece la Nota al testo relativa all'ed. critica citata (GIRALDI, Gli Ecatommiti, vol. III, pp. 2006-09).

63. Parafraso il titolo di un importante contributo di M. Pieri, La strategia edificante degli 'Ecatommiti', in «Esperienze letterarie», 3 (I978), pp. 43-74. 